\title{
Irremissible stimulate on 'Unified fixed point theorems in fuzzy metric spaces via common limit range property'
}

\author{
Antonio-Francisco Roldán-López-de-Hierro', Erdal Karapınar²,3 and Poom Kumam ${ }^{4}$
}

\author{
"Correspondence: \\ poom.kum@kmutt.ac.th \\ ${ }^{4}$ Department of Mathematics, \\ Faculty of Science, King Mongkut's \\ University of Technology Thonburi \\ (KMUTT), Bang Mod, Thrung Khru, \\ Bangkok, 10140, Thailand \\ Full list of author information is \\ available at the end of the article
}

\begin{abstract}
One of the goals of this short note is to alert researchers as regards some mistakes that appeared in a recent paper (Chauhan, Khan and Kumar in J. Inequal. Appl. 2013:182, 2013). This entails main proofs based on a false result, which invalidates all statements. We also give a complete revision of the antecedents of this work in order to find the main reasons of the mistakes. Finally, the main aim of this note is to propose a correct, more general version of the main theorems in the paper mentioned.
\end{abstract}

Keywords: fuzzy metric space; triangular norm; continuous t-norm

\section{Introduction}

In 2011, Sintunavarat and Kumam [1] first introduced the concept of 'common limit range property' (or (CLR) property) in metric spaces and fuzzy metric spaces and also improved the results of Mihet [2]. It is observed that the concept of (CLR) property never requires the condition of closedness of the subspace, while other properties such as the E.A. property requires this condition for the existence of the fixed point. Afterward, several mathematicians have proved common fixed point theorems in fuzzy metric spaces for different contractive conditions.

Very recently, Chauhan et al. announced in [3] a common fixed point theorem in the setting of fuzzy metric spaces for weakly compatible pairs $(A, S)$ and $(B, T)$ (where $A, S, B, T: X \rightarrow X$ are self-mappings on a fuzzy metric space $(X, M, *))$. This result involves the following notion.

Definition 1 Two pairs $(A, S)$ and $(B, T)$ of self-mappings of a fuzzy metric space $(X, M, *)$ are said to satisfy the common limit range property with respect to mappings $S$ and $T$ (briefly, $\left(C L R_{S T}\right)$ property), if there exist two sequences $\left\{x_{n}\right\},\left\{y_{n}\right\}$ in $X$ such that

$$
\lim _{n \rightarrow \infty} A x_{n}=\lim _{n \rightarrow \infty} S x_{n}=\lim _{n \rightarrow \infty} B x_{n}=\lim _{n \rightarrow \infty} T x_{n}=z
$$

where $z \in S(X) \cap T(X)$.

The contractivity property that the pairs $(A, S)$ and $(B, T)$ verify is the following one. 
Inequality (4.1) in [3]. There exists a constant $k \in(0,1)$ such that

$$
\phi\left(\begin{array}{c}
M(A x, B y, k t), M(S x, T y, t), M(A x, S x, t), \\
M(B y, T y, t), M(A x, T y, t), M(B y, S x, t)
\end{array}\right) \geq 0,
$$

for all $x, y \in X, t>0$ and $\phi \in \Phi_{6}$.

In that paper, $\Phi_{6}$ denoted the set of all continuous functions $\phi:[0,1]^{6} \rightarrow \mathbb{R}$ satisfying the conditions:

$\left(\phi_{1}\right) \phi$ is decreasing in $t_{2}, t_{3}, t_{4}, t_{5}$ and $t_{6}$.

$\left(\phi_{2}\right) \phi(u, v, v, v, v, v) \geq 0$ implies $u \geq v$, for all $u, v \in[0,1]$.

Using the previous preliminaries, Chauhan et al. announced the following result.

Theorem 2 (Chauhan et al. [3], Theorem 4.1) Let $(X, M, *)$ be a fuzzy metric space with

$$
t * t \geq t, \quad \text { for all } t \in[0,1] .
$$

Let $A, B, S$ and $T$ be mappings from $X$ into itself satisfying inequality (4.1). Suppose that the pairs $(A, S)$ and $(B, T)$ enjoy the $\left(C L R_{S T}\right)$ property. Then the pairs $(A, S)$ and $(B, T)$ have a coincidence point each. Moreover, $A, B, S$ and $T$ have a unique common fixed point provided both the pairs $(A, S)$ and $(B, T)$ are weakly compatible.

The proof given by the authors is decisively based on the following result.

Lemma 3 (Chauhan et al. [3], Lemma 2.2) Let $(X, M, *)$ be a fuzzy metric space with

$$
t * t \geq t, \quad \text { for all } t \in[0,1] .
$$

If there exists a constant $k \in(0,1)$ such that

$$
M(x, y, k t) \geq M(x, y, t), \quad \text { for all } x, y \in X
$$

then $x=y$.

However, Lemma 3 is wrong in two senses: firstly, it is incorrectly enunciated in [3] and, furthermore, it is false (therefore, it was not proved in [3]). As a consequence, all results in the mentioned paper were not correctly proved.

The main aim of this paper is to present alternative hypotheses to give validity to the main results in [3].

In this paper, we start giving a counterexample of Lemma 3 , showing why it is incorrectly enunciated. Then we investigate the antecedents of this mistake, and we show some other papers that presented the same mistake. In addition to this, we explain how hypothesis (1) is not interesting in the setting of fuzzy metric spaces, because it yields a very restricted kind of fuzzy metrics. Finally, we replace this hypothesis by a more convenient condition and we prove similar results to the ones presented in [3]. 


\section{Preliminaries}

Before presenting our main claims, we need to introduce some concepts and basic results. For instance, the notion of triangular norm plays a key role in different fields of study.

Definition 4 (Schweizer and Sklar [4]) A triangular norm $*$ (shortly $t$-norm) is a binary operation on the unit interval $[0,1]$ such that, for all $a, b, c, d \in[0,1]$, the following conditions are satisfied:

(i) $a *(b * c)=(a * b) * c$ (that is, $*$ is associative);

(ii) $a * b=b * a$ (that is, $*$ is commutative);

(iii) $a * 1=a$;

(iv) $a * b \leq c * d$ whenever $a \leq c$ and $b \leq d$.

A continuous t-norm is a $t$-norm which is also continuous as a mapping.

For instance, $t * s=\min (t, s)$, for all $t, s \in[0,1]$, is a well-known continuous $t$-norm, called the minimum $t$-norm. A continuous $t$-norm is one of the most important ingredients of the notion of fuzzy metric space. There are two definitions of the concept of fuzzy metric space that have attracted much attention in the last decades. Firstly, inspired by Zadeh fuzzy sets [5] and the concept of Menger probabilistic metric space [6], in 1975, Kramosil and Michálek [7] presented a new model that lets one apply fuzzy behavior to real situations and they defined a Hausdorff topology on these spaces.

Definition 5 (Kramosil and Michálek [7]) A 3-tuple $(X, M, *)$ is said to be a fuzzy metric space if $X$ is an arbitrary set, $*$ is a continuous $t$-norm and $M$ is a fuzzy set on $X \times X \times[0, \infty)$ satisfying the following conditions: for all $x, y, z \in X, t, s \geq 0$,

$(\mathrm{KM}-1) \quad M(x, y, 0)=0$,

(KM-2) $M(x, y, t)=1$ if and only if $x=y$,

(KM-3) $M(x, y, t)=M(y, x, t)$,

(KM-4) $M(x, z, t+s) \geq M(x, y, t) * M(y, z, s)$,

$(\mathrm{KM}-5) M(x, y, \cdot):[0, \infty) \rightarrow[0,1]$ is left-continuous.

Later, George and Veeramani [8] slightly modified the concept of fuzzy metric space introduced by Kramosil and Michálek, defined a Hausdorff topology, and proved some known results including Baire's theorem (see also [9]).

Definition 6 (George and Veeramani [8]) A 3-tuple $(X, M, *)$ is said to be a fuzzy metric space if $X$ is an arbitrary set, $*$ is a continuous $t$-norm, and $M$ is a fuzzy set on $X \times X \times$ $(0, \infty)$ satisfying the following conditions: for all $x, y, z \in X, t, s>0$,

$(\mathrm{FM}-1) M(x, y, t)>0$,

(FM-2) $M(x, y, t)=1$ if and only if $x=y$,

(FM-3) $M(x, y, t)=M(y, x, t)$,

(FM-4) $M(x, z, t+s) \geq M(x, y, t) * M(y, z, s)$,

$(\mathrm{FM}-5) M(x, y, \cdot):(0, \infty) \rightarrow(0,1]$ is continuous.

Then $M$ is called a fuzzy metric on $X$ whereas $M(x, y, t)$ denotes the degree of nearness between $x$ and $y$ with respect to $t$.

The following result holds in both classes of fuzzy metric spaces. 
Lemma 7 If $(X, M, *)$ is a fuzzy metric space and $x, y \in X$, then the mapping $M(x, y, \cdot)$ is non-decreasing.

\section{A counterexample of Lemma 3}

First of all, we point out that Lemma 3 is incorrectly enunciated because condition (2) should be: 'if $x, y \in X$ are given points such that there exists $k \in(0,1)$ verifying

$$
M(x, y, k t) \geq M(x, y, t), \quad \text { for all } t>0,
$$

then $x=y$ '. However, as we show in the following example, the condition ' $t * t \geq t$, for all $t \in[0,1]$ ' is not strong enough to guarantee that the thesis of Lemma 3 holds. Notice that the following example is valid for fuzzy metric spaces in the sense of both Definitions 5 and 6 .

Example 8 Let $X$ be a set containing, at least, two different points, let $*$ be a continuous $t$-norm and let $c \in] 0,1[$ be arbitrary. Define $M: X \times X \times[0, \infty) \rightarrow[0,1]$, for all $x, y \in X$ and $t \geq 0$, by

$$
M(x, y, t)= \begin{cases}0, & \text { if } t=0 \\ 1, & \text { if } t>0 \text { and } x=y \\ c, & \text { if } t>0 \text { and } x \neq y\end{cases}
$$

Then $(X, M, *)$ is a fuzzy metric space in the sense of Kramosil and Michálek. Furthermore, the restriction of $M$ to $X \times X \times(0, \infty)$ defines a fuzzy metric on $X$ in the sense of George and Veeramani. In addition to this, condition (2) holds, for all $k \in(0,1)$ and all $x, y \in X$ even though $x \neq y$.

\section{A correct version of Lemma 3}

Before explaining the antecedents of the mistake that appears in Lemma 3, we show how other authors have successfully used this property in the past. The main reason why the fuzzy metric given in Example 8 does not verify Lemma 3 is the fact that $M(x, y, \cdot)$ is constant on $(0, \infty)$. Therefore, it verifies the condition

$$
\lim _{t \rightarrow \infty} M(x, y, t)=c<1 \quad \text { if } x \neq y .
$$

In the sequel, unless it is stated otherwise, we will only refer to fuzzy metric spaces in the sense of George and Veeramani (but similar properties are valid in Kramosil and Michálek's spaces).

Property (3) is not coherent with the idea that $M(x, y, t)$ denotes the degree of nearness between $x$ and $y$ with respect to $t$ and it leads to no very good properties. In order to avoid the condition (3), it is usual to consider additionally the axiom.

(FM-6) For all $x, y \in X$, we have $\lim _{t \rightarrow \infty} M(x, y, t)=1$.

This is the case of [10-14]. In this kind of space, the following result is well known.

Lemma 9 Let $(X, M, *)$ be a fuzzy metric space verifying (FM-6) and let $x, y \in X$. If there exists $k \in[0,1)$ such that $M(x, y, k t) \geq M(x, y, t)$, for all $t>0$, then $x=y$. 
As we have just seen in Example 8, this property is clearly false if the fuzzy metric does not satisfy axiom (FM-6).

Proof We prove that $M(x, y, \cdot)$ is constant in $(0, \infty)$. Let $t, s \in(0, \infty)$ be such that $s \leq t$. As $\left\{k^{n}\right\} \rightarrow 0$, we can find $n \in \mathbb{N}$ such that $k^{n} t \leq s \leq t$. As $M(x, y, \cdot)$ is non-decreasing, $M\left(x, y, k^{n} t\right) \leq M(x, y, s) \leq M(x, y, t)$. Using the hypothesis,

$$
M\left(x, y, k^{n} t\right) \geq M\left(x, y, k^{n-1} t\right) \geq \cdots \geq M(x, y, k t) \geq M(x, y, t) .
$$

Therefore, $M\left(x, y, k^{n} t\right) \leq M(x, y, s) \leq M(x, y, t) \leq M\left(x, y, k^{n} t\right)$ which proves that $M(x, y, t)=$ $M(x, y, s)$. Thus, $M(x, y, \cdot)$ is constant in $(0, \infty)$. Using axiom (FM-6), $M(x, y, t)=$ $\lim _{s \rightarrow \infty} M(x, y, s)=1$, for all $t>0$, and using axiom (FM-2) we conclude that $x=y$.

As we have just showed, the hypothesis ' $t * t \geq t$, for all $t \in[0,1]$ ' (which we can find in Lemma 3 ) is not strong enough to guarantee that the thesis of Lemma 3 holds. Moreover, we are going to prove that it is a very restrictive hypothesis, because there is an unique example of $t$-norm (not necessarily continuous) verifying this property.

Lemma $10([4,15])$ The only t-norm $*$ verifying

$$
t * t \geq t, \quad \text { for all } t \in[0,1]
$$

is the minimum $t$-norm.

Proof Let $t, s \in[0,1]$ be arbitrary. Without loss of generality, we can suppose that $s \leq t$. Since $0 \leq s \leq t \leq 1$ and $*$ is non-decreasing on each argument, it follows that $s * s \leq s * t \leq$ $s * 1=s$. Combining these inequalities with the assumption $s \leq s * s$, we derive $s \leq s * t \leq s$. Therefore, $s * t=s=\min (s, t)$, for all $t, s \in[0,1]$.

In both senses, Lemma 3 is wrong and, therefore, researchers always try to avoid condition (4) in the setting of fuzzy metric spaces.

\section{The antecedents of Lemma 3}

To better understand our way to improve the results in [3], we must review some previous papers, paying especially attention to Lemma 9. In [3], Remark 4.2, the authors announced that their results improved some theorems by other researchers. For instance, they mentioned Rao et al. [16, Theorem 3.1]. In this paper, we can find a version of Lemma 9 avoiding the hypothesis (FM-6) (see [16, Lemma 1.7]). Therefore, it is false. Later, Rao et al. advised that they had found a gap in a result by Cho [17], which used condition (4). Unfortunately, hypothesis (4) was not supposed on any main result in [17]. In that paper, we can also find Lemma 10, but there is also included a version of Lemma 9 avoiding the hypothesis (FM-6) (see [17, Lemma 2.7]), which is false. Based on Cho's paper, Rao et al. proved some results in which we can found condition (4) as a hypothesis. Therefore, their results are very restrictive because they are only valid in fuzzy metric spaces under the minimum $t$-norm. Furthermore, their proofs are not valid because they are based on a version of Lemma 9 without axiom (FM-6). 
In [3], the authors also announced that their results also improved some theorem by Aalam et al. [18]. In that paper, we can also found a version of Lemma 9 avoiding the hypothesis (FM-6) (see [18, Lemma 1.1]), which is false. Therefore, the proofs of their main results are not correct. Precisely, at this point, we can find the reason of all gaps: these authors referred to Mishra et al. [10] to include this lemma in their preliminaries, but they did not realize that Mishra et al.'s paper is based on Kramosil and Michálek's fuzzy metric spaces that, additionally, verify axiom (FM-6).

Taking into account these preliminaries and trying to improve different papers, Chauhan et al. collected in [3] two gaps: from [18], they assumed that Lemma 9 holds, avoiding the hypothesis (FM-6), which is false; and from [16], they supposed condition (4) in their main results, which is not necessary.

\section{Correct versions of results given in [3]}

To sum up the previous considerations, we must remark the following facts.

- On the one hand, the proofs given by the authors of the main results in [3] are incorrect because they are based on Lemma 3, which is false. We have investigated the origin of this mistake.

- On the other hand, we advise that the only $t$-norm (not necessarily continuous) that verifies property (4) is the minimum $t$-norm, which is a very restrictive hypothesis.

All researchers in this field try to avoid it because it produces no very general results.

The main aim of this paper is to provide different conditions to give validity to the main results given in [3]. For instance, in order to overcome the mentioned drawbacks, we propose to replace the condition $t * t \geq t$, for all $t \in[0,1]$, using axiom (FM-6). In this case, the following result holds.

Theorem 11 The main results in [3] are valid if we replace the condition ' $t * t \geq t$ for all $t \in[0,1]$ 'by axiom (FM-6).

In fact, the same proofs, based on Lemma 9, can be followed through point by point.

Competing interests

The authors declare that they have no competing interests.

Authors' contributions

All authors contributed equally and significantly in writing this paper. All authors read and approved the final manuscript.

Author details

${ }^{1}$ Department of Mathematics, University of Jaén, Campus Las Lagunillas, s/n, Jaén, 23071, Spain. ²Department of Mathematics, Atilim University, Incek, Ankara, 06836, Turkey. ${ }^{3}$ Nonlinear Analysis and Applied Mathematics Research Group (NAAM), King Abdulaziz University, Jeddah, Saudi Arabia. ${ }^{4}$ Department of Mathematics, Faculty of Science, King Mongkut's University of Technology Thonburi (KMUTT), Bang Mod, Thrung Khru, Bangkok, 10140, Thailand.

\section{Acknowledgements}

The first author has been partially supported by Junta de Andalucía by project FQM-268 of the Andalusian CICYE. The third author was supported by the Higher Education Research Promotion and National Research University Project of Thailand, Office of the Higher Education Commission (NRU57).

\section{Received: 4 March 2014 Accepted: 6 June 2014 Published: 22 Jul 2014}

\section{References}

1. Sintunavarat, W, Kumam, P: Common fixed point theorems for a pair of weakly compatible mappings in fuzzy metric spaces. J. Appl. Math. 2011, Article ID 637958 (2011)

2. Mihet, M: Fixed point theorems in fuzzy metric spaces using property E. A. Nonlinear Anal. 73, 2184-2188 (2010)

3. Chauhan, S, Khan, MA, Kumar, S: Unified fixed point theorems in fuzzy metric spaces via common limit range property. J. Inequal. Appl. 2013, Article ID 182 (2013) 
4. Schweizer, B, Sklar, A: Probabilistic Metric Spaces. Dover, New York (2005)

5. Zadeh, LA: Fuzzy sets. Inf. Control 8, 338-353 (1965)

6. Menger, K: Statistical metrics. Proc. Natl. Acad. Sci. USA 28, 535-537 (1942)

7. Kramosil, I, Michálek, J: Fuzzy metrics and statistical metric spaces. Kybernetika 11, 336-344 (1975)

8. George, A, Veeramani, P: On some results in fuzzy metric spaces. Fuzzy Sets Syst. 64, 395-399 (1994)

9. Roldán, A, Martínez-Moreno, J, Roldán, C: On interrelationships between fuzzy metric structures. Iran. J. Fuzzy Syst. 10(2), 133-150 (2013)

10. Mishra, SN, Sharma, N, Singh, SL: Common fixed point of maps on fuzzy metric spaces. Int. J. Math. Math. Sci. 17, 253-258 (1994)

11. Cho, YJ: Fixed points in fuzzy metric spaces. J. Fuzzy Math. 5(4), 949-962 (1997)

12. Sharma, S: Common fixed point theorems in fuzzy metric spaces. Fuzzy Sets Syst. 127, 345-352 (2002)

13. Liu, Y, Li, Z: Coincidence point theorems in probabilistic and fuzzy metric spaces. Fuzzy Sets Syst. 158, 58-70 (2007)

14. Roldán, A, Martínez-Moreno, J, Roldán, C, Cho, YJ: Multidimensional coincidence point results for compatible mappings in partially ordered fuzzy metric spaces. Fuzzy Sets Syst. (2013). doi:10.1016/j.fss.2013.10.009

15. Klement, EP, Mesiar, R, Pap, E: Triangular Norms. Kluwer Academic, Dordrecht (2000)

16. Rao, KPR, Babu, GVR, Fisher, B: Common fixed point theorems in fuzzy metric spaces under implicit relations. Hacet. J. Math. Stat. 37(2), 97-106 (2008)

17. Cho, SH: On common fixed points in fuzzy metric spaces. Int. Math. Forum 1(10), 471-479 (2006)

18. Aalam, I, Kumar, S, Pant, BD: A common fixed point theorem in fuzzy metric space. Bull. Math. Anal. Appl. 2(4), 76-82 (2010)

10.1186/1029-242X-2014-257

Cite this article as: Roldán-López-de-Hierro et al.: Irremissible stimulate on 'Unified fixed point theorems in fuzzy metric spaces via common limit range property'. Journal of Inequalities and Applications 2014, 2014:257

\section{Submit your manuscript to a SpringerOpen ${ }^{\circ}$ journal and benefit from:}

- Convenient online submission

- Rigorous peer review

- Immediate publication on acceptance

- Open access: articles freely available online

- High visibility within the field

- Retaining the copyright to your article 